\title{
Performance Models for Sidelobe Constrained Signal Design with Eigen-Basis Formulation
}

\author{
Aaron M. Jones \\ Sensors Directorate \\ AFRL: RF Exploitation Branch \\ Wright Patterson AFB, Ohio 45433
}

\author{
Brian D. Rigling \\ Department of Electrical Engineering \\ Wright State University \\ Dayton, Ohio 45435
}

\author{
Muralidhar Rangaswamy \\ Sensors Directorate \\ AFRL: RF Exploitation Branch \\ Wright Patterson AFB, Ohio 45433
}

\begin{abstract}
Understanding the consequences of applying constraints in arbitrary waveform design can benefit the decision making process of an adaptive system by providing insight into selection of the transmit signal. In this paper, we discuss the role of the integrated sidelobe (ISL) parameter for adaptive radar signal design as it pertains to signal-to-interference-ratio (SINR) performance. We do so by undertaking a design methodology that limits the basis dimension of the waveform while placing a finite energy constraint on the optimal ISL solution. We further extend the analysis by developing performance models for the integrated sidelobe metrics corresponding SINR degradation, from optimal. Included are Monte Carlo simulation trials designed to measure the impact of ISL on SINR.
\end{abstract}

\section{INTRODUCTION AND Motivation}

The optimal waveform design solution for signal-tointerference-ratio is the Rayleigh solution or the eigen-vector corresponding to the minimum eigenvalue of the noise and interference covariance matrix [1]. However, this waveform is unlikely to exhibit low integrated sidelobes, low peak sidelobe ratio (PSLR), or efficiently use the available power resource. Thus, constraints are placed on the signal design to improve the practicality for radar. For example, we desire low integrated sidelobes to prevent masking of low SINR targets while low peak sidelobes reduces false alarms.

Results pertaining to the impact of each of these constraints with respect to SINR performance were reported in [2]. Additionally, in-depth analysis and performance models of the modulus constraint are shown in [3] and we note the same signal design approach is employed in this paper, albeit for a different signal parameter, i.e. ISL. These considerations significantly impact waveform design for cognitive and/or fully adaptive radar (FAR), especially when rapid waveform refinement is called for in a dynamically changing environment.

The integrated sidelobe constraint as a signal design criteria has been studied in great depth [4]-[9] and we note its important role in multiple radar functions, including reducing the number of false alarms, improving multiple target resolution and efficient spreading of the energy contained within the signal. Others have investigated performance models for similar metrics, integrated sidelobe ratio and peak-sidelobe ratio but limited the data models of noise and interference modeled to Bernoulli distributions [10], [11].

The literature also mentions several design methodologies for limiting sidelobe levels, including; periodic autocorrelation function technique that has zero sidelobes [12], and similarly a cyclic technique PeCAN [8], [13] or a competing technique Signal Waveform's Optimal Under Restriction Design for Active Sensing (SWORD) [14], among others. However, the progressive consequence to SINR when synthesizing waveforms with these techniques is unclear.

In this paper, a technique where the optimized signal is a subset of a linear combination of eigenvectors of the noise and interference covariance matrix is employed, also seen in [2]. We point out that any waveform may be viewed as a linear combination of the eigenvectors of this matrix but, what is uncertain, is how the signal interaction with the noise subspace degrades SINR performance. For the Rayleigh solution, the waveform is defined by a single eigenvector, but applying waveform constraints implies additional eigendimensions must be spanned. This is problematic, for as the design leaks, or projects, waveform energy into the higher noise and interference subspace, SINR will invariably degrade.

Defining how constraints affect SINR performance can improve the decision making process of such an adaptive system. For example, one might sacrifice peak-to-average-power (PAPR) for ISL performance in a high SINR environment to resolve near-in targets. Conversely, the most efficient use of power might be necessary, limiting the degrees of freedom for ISL performance. This paper will give insight into the behavior of the trade-space between the competing signal traits of SINR and ISL levels. Certainly, the PAPR of the signal should be considered in practice, however, we focus our concern on the ISL constraint for this paper to better understand its role in signal performance.

In Section II, we discuss fundamentals and theory used to illustrate our work. Section III outlines the problem statement and technical approach, and in Section IV we give empirical results derived from Monte Carlo computer simulations using MATLAB [15]. We state conclusions, insights and intended future work in Section V. The appendix contains the associated gradient and Hessian of the primary cost function and a discussion of non-convexity.

Column vectors are denoted as underlined lowercase letters. The $n^{\text {th }}$ entry of a column vector $\underline{\mathrm{s}}$ is denoted $\underline{\mathrm{s}}_{n}$. Matrices are shown using boldface capital letters. Scalar quantities are given as capital letters. $\mathbb{R}\{\cdot\}$ and $\mathbb{I}\{\cdot\}$ represent the real and imaginary components of a complex argument, respectively. 
The Hermitian operator is given as $(\cdot)^{H}$, while the expectation operator is $\mathbf{E}\{\cdot\}$. The $\mathrm{L}_{2}$ norm is represented as $\|\cdot\|_{2}$ and the optimal solution is denoted as $(\cdot)^{*}$. Finally, the partial derivative operator is indicated by the $\partial$ identifier with the gradient as $\nabla$ and the Hessian denoted $\mathcal{H}$.

\section{PRELIMINARIES}

We define the transmit signal $\underline{\mathrm{s}} \in \mathbb{C}^{M \times 1}$, and the unprocessed received signal

$$
\underline{\mathrm{y}}=\underline{\mathrm{s}}+\underline{\mathrm{n}},
$$

where $\underline{\mathrm{n}} \in \mathbb{C}^{M \times 1}$ is interference and wide sense stationary additive noise. For the ideal case of known range, we project the recieved signal onto the transmit subspace giving

$$
\underline{\mathrm{s}}^{H} \mathrm{y}=\underline{\mathrm{s}}^{H} \underline{\mathrm{s}}+\underline{\mathrm{s}}^{H} \underline{\mathrm{n}},
$$

and assume a power constrained transmit signal enforced by the constraint $\|\underline{s}\|_{2}^{2}=1$ (choosing 1 for convenience). The expected SINR is then

$$
\mathrm{E}\{\mathrm{SINR}\}=\frac{\mathrm{E}\left\{\underline{\mathrm{s}}^{H} \underline{\mathrm{s}}^{H} \underline{\mathrm{s}}\right\}}{\mathrm{E}\left\{\underline{\mathrm{s}}^{H} \underline{\mathrm{nn}}^{H} \underline{\mathrm{s}}\right\}}=\frac{1}{\underline{\mathrm{s}}^{H} \mathbf{K} \underline{\mathrm{s}}},
$$

where $\mathbf{K} \in \mathbb{C}^{M \times M}$ is the clairvoyant interference and noise covariance matrix. For our analysis, the covariance is assumed known but in practice can be well estimated using a multitude of techniques with necessary sample size $(\approx 2 \mathrm{M})$ conforming to Brennan's rule [16].

Consequently, quadratic optimizations, where the objective function is seen in the denominator of (3),

$$
\min _{\underline{\mathbf{s}}} \underline{\mathrm{s}}^{H} \mathbf{K} \underline{\mathbf{s}}, \text { s. t. }\|\underline{\mathbf{s}}\|_{2}^{2}=1 \text {, }
$$

often appear in radar waveform design applications. Again, $\underline{\mathbf{S}}$ is the waveform to be designed and $\mathbf{K}$ is Hermitian positive definite, and represents the clutter, interference and noise covariance matrix capturing the second-order statistical properties. Examples of this problem form are seen in [17], [18].

For our purposes, we express the designed waveform as a linear combination of an ordered subset of the eigenvectors of the interference and noise covariance matrix. For an eigen decomposition

$$
\mathbf{K}=\mathbf{V}^{H} \mathbf{\Lambda} \mathbf{V}
$$

where $\mathbf{V}=\left[\underline{\mathrm{v}}_{1} \cdots \underline{\mathrm{v}}_{M}\right]$ and $\boldsymbol{\Lambda}=\operatorname{diag}\left\{\lambda_{1} \cdots \lambda_{M}\right\}$ such that $0<\lambda_{1} \leq \lambda_{2} \leq \cdots \leq \lambda_{M}$, we set out to use the smallest number of eigenvectors from the low-noise subspace to represent the signal. The waveform is represented as

$$
\underline{\mathrm{s}}=\sum_{k=1}^{N} \alpha_{k} \underline{\mathrm{v}}_{k}
$$

where $\alpha_{k} \in \mathbb{C}^{1 \times 1}$ is the $k^{\text {th }}$ eigenvector weight, and $N \leq$ $M$ eigenvectors comprise the support of $\underline{s}$ [19], [20]. In our analysis, we report the results over the entire eigen-basis to form a performance model of ISL and SINR as a function of the basis dimension of the signal, i.e., $N$.
The ISL is defined as the total energy of the sidelobes of the waveform's autocorrelation sequence (ACS). However, we are able to exploit the symmetry of the ACS to simplify and compute this quantity as

$$
\mathrm{J}_{\mathrm{ISL}}(\underline{\mathrm{s}})=\sum_{k=1}^{M-1}\left|\underline{\mathrm{s}}^{H} \mathbf{E}_{\mathbf{k} \underline{\mathrm{s}}}\right|^{2},
$$

where $\mathbf{E}_{k} \in \mathbb{R}^{M \times M}$ is a zero matrix with 1's on the $k^{t h}$ upper superdiagonal. This formulation assumes a single lag mainlobe width to compute the energy, a possible over simplification in some cases.

Also, and pragmatically, a finite energy limit is placed on the signal. This is the case in practice and the convex constraint on the $\mathrm{L}_{2}$ norm $\left(\|\underline{\mathrm{s}}\|_{2}^{2}=1\right)$ imposes this restriction in the analysis. While the value can be chosen arbitrarily, we have set it equal to unity, for ease of use.

To generalize the data representation we use a well known result in random matrix theory that states, in the limit of large data, that the statistical behavior of eigenvalues of large random Hermitian matrices, under the assumption of a fullrank covariance with $\sum_{k=1}^{M} \lambda_{k}=M$ (equivalent to assuming constant noise power), adheres to the following, simplified, Marčenko-Pastur (MP) density when the matrix is square [21]

$$
p_{\Lambda}(\lambda)=\frac{\sqrt{4 \lambda-\lambda^{2}}}{2 \lambda}, \lambda \in(0,4] .
$$

This data model is unique due to the closed form expression of the eigen-value distribution, allowing simplified development of the E\{SINR $\}$ model. See [3] for depiction of this density and the corresponding cumulative distribution function. Figure 1 shows two instantiations of the PSD for this data model.

Additionally, the MP density allows for a convenient mathematical model of the eigenvalues and we define the covariance

$$
\mathbf{K}=\frac{1}{M} \mathbf{Z} \mathbf{Z}^{H},
$$

where $\mathbf{Z}_{i j}$ are $\mathbb{C N}(0,1)$.

Lastly, the expected SINR of the output of a matched filter, with fixed energy constraint $\left(\|\underline{s}\|_{2}^{2}=1\right)$, can be expressed as a linear combination of weighted eigenvalues,

$$
\mathrm{E}\{\mathrm{SINR}\}=\frac{1}{\sum_{k=1}^{N \leq M}\left|\underline{\alpha}_{k}\right|^{2} \lambda_{k}} .
$$

In our case, the eigenvalues are computed in closed-form via the MP distribution and are considered known for a length $M$ signal. The problem becomes a matter of selecting the weights to compute SINR, where, for example, the "best" SINR corresponds to $\underline{\alpha}_{1}=1$ and all others as zero. The "worst" performance would be inclusion of only the $N^{\text {th }}$ eigenvalue while an average would put an equal weight on each of the $N$ values.

\section{Problem Formulation}

Employing the technique in [3] allows us to investigate the degradation in SINR while explicitly designing the signal to 


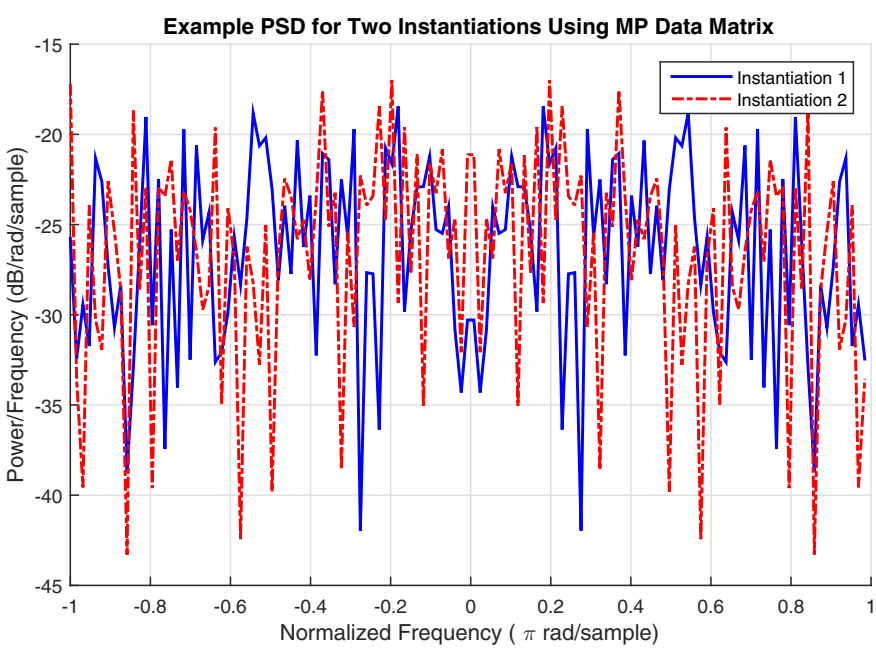

Fig. 1. Example power spectral density of two instantiations of the interference and noise spectrum when employing the MP data generation technique in (9) for a length $M=64$ signal.

minimize its energy outside the mainlobe. Using the definition in (7) of the ACS, we define our optimization problem as

$$
\min _{\underline{\alpha}} \sum_{k=1}^{M-1}\left|\underline{\mathbf{s}}{ }^{H} \mathbf{E}_{\mathbf{k}} \underline{\underline{s}}\right|^{2}, \text { s. t. }\|\underline{\mathbf{s}}\|_{2}^{2}=1 \text {. }
$$

A closed-form representation for $\sum_{k=1}^{M-1}\left|\underline{\mathbf{s}}^{H} \mathbf{E}_{\mathbf{k}} \underline{\mathrm{s}}\right|^{2}$ in terms of specific eigenvectors $\underline{\mathrm{v}}$ corresponding to a single instant ation of noise and interference is mathematically intractabl We note the eigenvectors cannot be represented by a randor variable (due to the norm restriction) nor do they offer an favorable structure in regards to the ACS, that we are awar of. Thus, the expected value cannot be found analytically an we are forced to develop an empirical model that accuratel represents the average.

\section{A. Initialization}

In [2] it is shown (11) is non-convex. This is problematic i the sense that a global critical point is not guaranteed, and th solution will, most likely, be highly dependent on the initi: submission to the solver. For our purposes, it is important $t$ determine the degradation of SINR from optimal as we includ. additional eigenvectors to minimize ISL. It is appropriate then to initialize with the optimal waveform (eigenvector associated with minimum eigenvalue) and place no emphasis with other

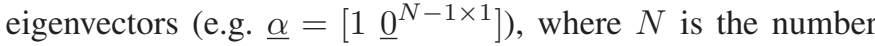
of eigenvectors used in the design.

\section{B. ISL Performance}

Intuitively, it is understood that minimizing the sidelobes whitens the waveforms spectral properties. However, to understand the behavior of the cost function, when the MP data matrix is used, we compute the average Wiener Entropy (i.e, spectral flatness measure (SFM)) of the eigenvectors of the interference and noise covariance matrix as well as the waveform itself over many Monte Carlo trials. It is computed as

$$
\mathrm{E}\{\mathrm{SFM}\}=\mathrm{E}\left\{\frac{\exp \frac{1}{M} \sum_{m=1}^{M} \ln (\operatorname{PSD}(m))}{\frac{1}{M} \sum_{m=1}^{M} \operatorname{PSD}(m)}\right\},
$$

or simply, the ratio of the PSD geometric mean to its arithmetic mean where a value of 1 would imply a spectrally flat PSD [22]. Without giving full details on the simulation (see section IV), we show in Figure 2 the computed $E\{S F M\}$ for two signals: (1) the designed waveform, and (2) the raw data or eigenvectors. We notice immediately the $\mathrm{E}\{\mathrm{SFM}\}$ of the eigenvectors remains relatively constant, implying no change in the spectral properties across the eigen-basis. For the signal $\mathrm{E}\{\mathrm{SFM}\}$, the analysis yields three distinct zones that can be tied the ISL performance (overlaid). We classify the zones in the following manner:

- Zone 1. Exponential increase in SFM, implies exponential decrease in ISL

- Zone 2. Linear increase in SFM, implies linear decrease in ISL

- Zone 3. Flat SFM, implies no ISL improvement

This is an important observation and if identification of the transitions can be defined for arbitrary signal and data models, this result would provide useful information in an adaptive system deciding where to place emphasis with the available degrees of freedom.

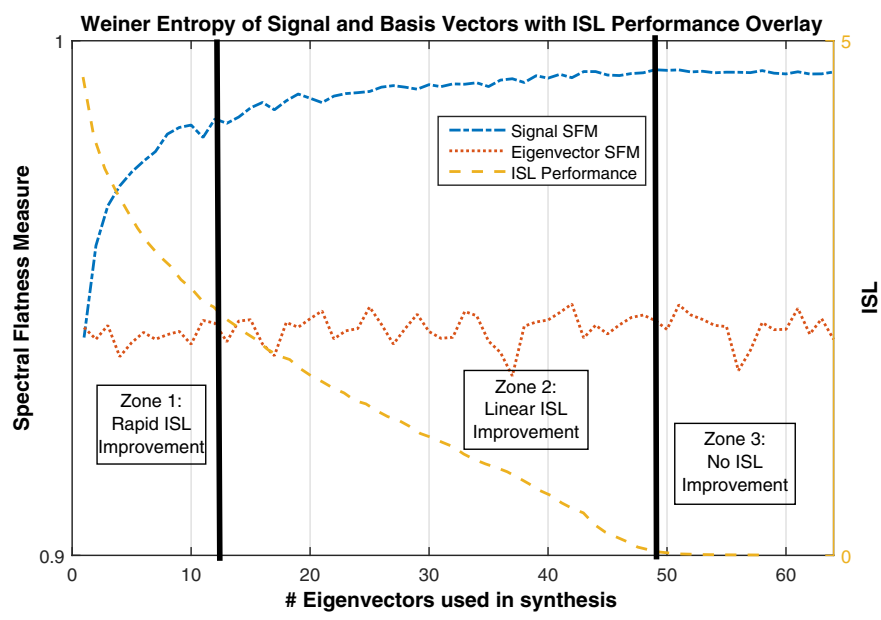

Fig. 2. Analysis of the spectral flatness measure of the eigen-basis, designed signal and how it compares to the ISL performance when employing the MP data generation technique in (9) for a length $M=64$ signal.

\section{SINR Performance Model}

Since the MP asymptotic distribution allows computation of an eigen-spectrum for a large matrix, whose column dimension corresponds to signal length, we consider the eigenvalues, $\lambda_{k}$ to be deterministic, and have modeled for the optimal weights $\underline{\alpha}^{*}$. It is then a straightforward process to calculate $\mathrm{E}\left\{\operatorname{SINR}\left(\underline{s}^{*}\right)\right\}$ under these assumptions. Applying the inverse transform sampling method to (8), a closed form representation 
of cumulative $\mathrm{CDF}$, thus a mechanism to compute the expected eigenvalues is possible. We can define the CDF as

$$
\begin{aligned}
\mathcal{M}(\lambda) & =\int_{0}^{\lambda} p_{\Lambda}(z) d z \\
& =\frac{1}{2 \pi}\left(\sqrt{4 \lambda-\lambda^{2}}-2 \arcsin \frac{4-2 \lambda}{4}\right)+\frac{1}{2},
\end{aligned}
$$

and compute the inverse survival function, $\mathcal{Z}(\lambda)$ to describe the eigen-spectrum for discrete, $\lambda$ as

$$
\mathcal{Z}(\lambda)=\frac{2}{\pi}\left(2 \arcsin \frac{4-2 \lambda}{4}+\pi-\sqrt{4 \lambda-\lambda^{2}}\right) .
$$

Shown in Fig. 3 is the MP eigen-spectrum $\mathcal{Z}(\lambda)$ as well as sample eigen-spectrum from 25 random instantiations.

Letting the magnitude of the weights be model by an exponential decay function $\left|\underline{\bar{\alpha}}_{N}(k)\right|=\frac{\exp \left\{-\frac{k}{N}\right\}}{\left\|\exp \left\{-\frac{k}{N}\right\}\right\|_{2}}$. We now write the $\operatorname{E}\left\{\operatorname{SINR}\left(\underline{s}^{*}\right)\right\}$ as

$$
\mathrm{E}\left\{\operatorname{SINR}\left(\underline{s}^{*}\right)\right\} \cong \frac{1}{\sum_{k=1}^{N \leq M}\left|\underline{\bar{\alpha}}_{N}(k)\right|^{2} \mathcal{Z}(k)},
$$

which allows for a simple representation of the consequences to SINR as the ISL tightens and forces a larger design space.

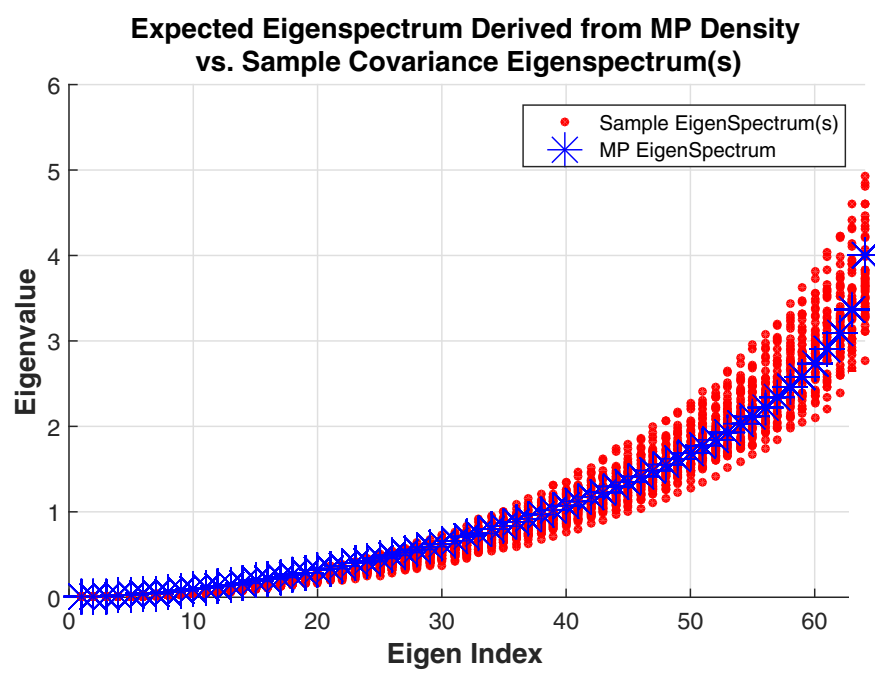

Fig. 3. Asymptotic Marčenko-Pastur eigen-spectum, derived from the dens in (8) with sample covariance eigen-spectrums generated from 25 rand instantiations of the data matrix for $M=64$, for comparison.

\section{Simulations}

The experimental approach shows the effect of the wav form basis dimension on the ISL objective and SINR degrad tion, from optimal, via computer simulations. The covariance matrix is assumed known and the eigen-spectrum represented by the MP asymptotic conclusion. We also hold transmit and noise power constant (and unit-less) throughout, and report the mean values derived from the MC trials.

The MATLAB software package, including the Optimization Toolbox, [15] was used to generate data, charts and perform the optimization routines. Algorithm 1 describes the process performed to generate the numerical results.

In Figure 4, the $\sum_{k=1}^{M-1}\left|\underline{\mathrm{s}}^{H} \mathbf{E}_{\mathbf{k}} \underline{\mathbf{s}}\right|^{2}$ as a function of the basis

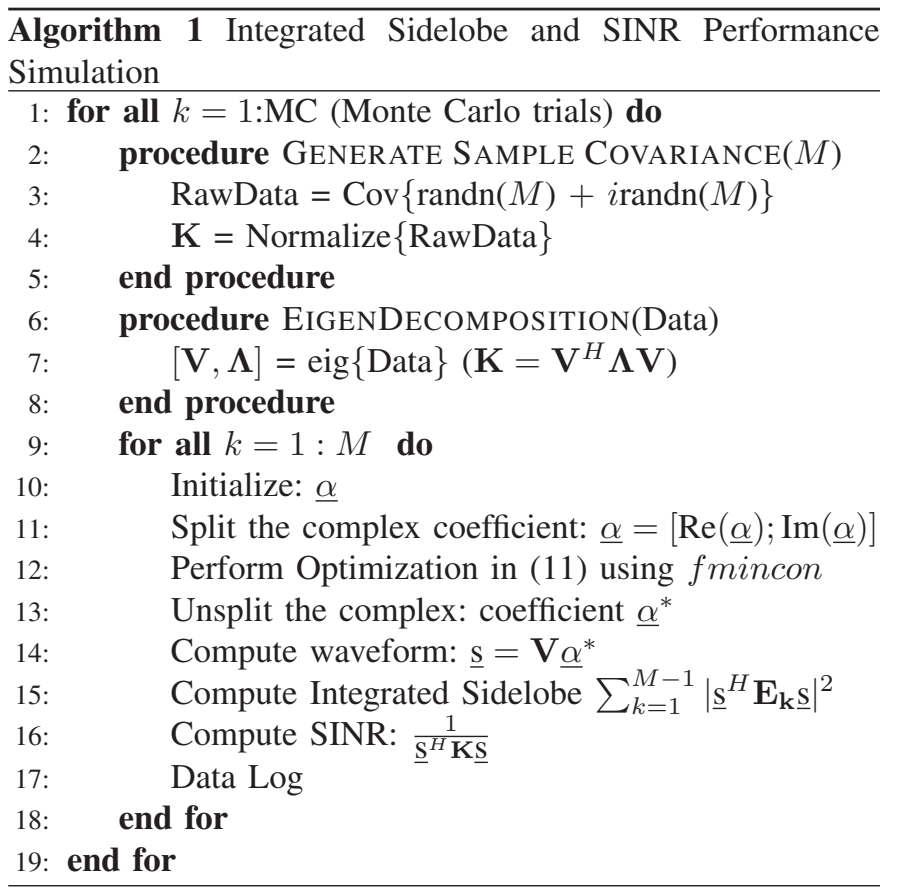

$(N)$ dimension is reported. The chart also gives the overall lowest (best) and highest (worst) calculated ISL. In the case of the worst performance, the solution is trapped in a suboptimal local minimum. To escape, or achieve a different result, a different initialization would need to be investigated.

Figure 5 shows the average SINR degradation, from

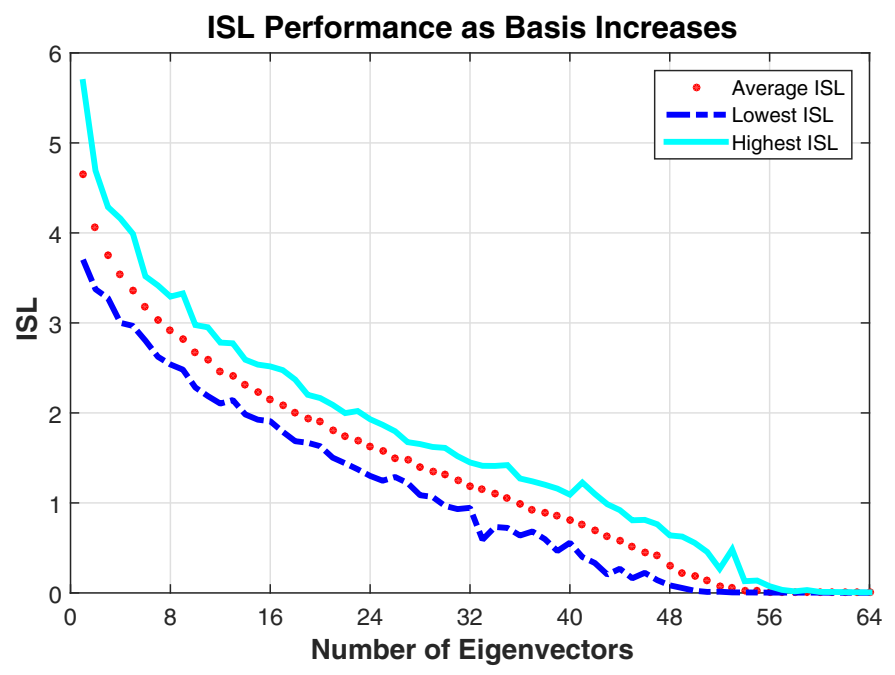

Fig. 4. ISL performance as a function of eigen-basis for 100 Monte Carlo trials, $M=64$. Included are also the lowest and highest computed sidelobe values for all simulations.

optimal. We note the large dynamic range is due to the minimum eigenvalue being, approximately, zero. Interestingly, the SINR of the highest ISL solution outperforms the average 


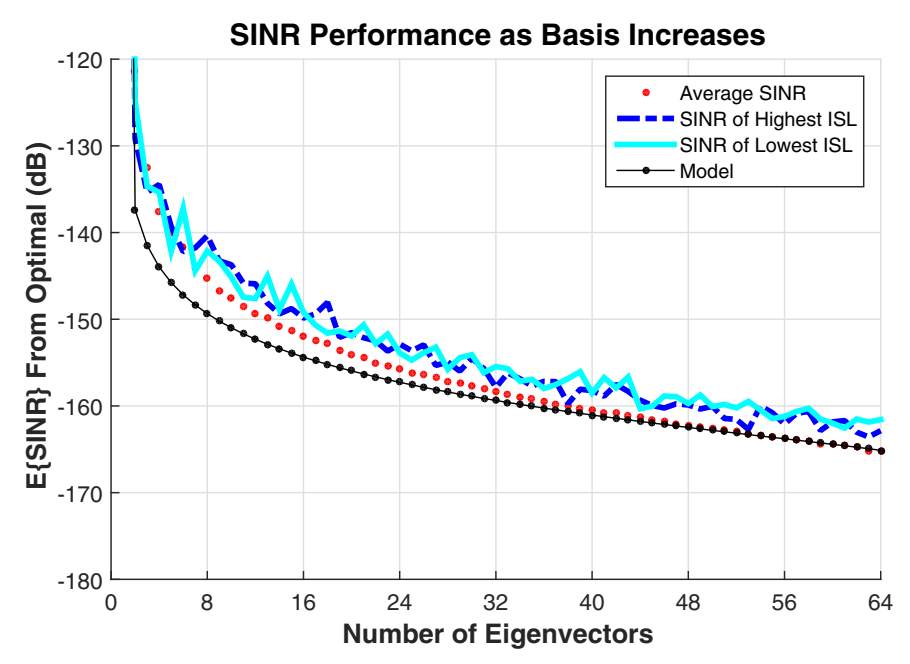

Fig. 5. SINR performance as the signal basis dimension increases for 100 Monte Carlo trials and $M=64$. Included in the figure are the average SINR, SINR of the lowest and highest ISL and the model using equation (15).

design, suggesting a more complicated relationship between ISL and SINR, possibly attributable to the spectral whiteness of the design, not necessarily just the dimension of the basis.

Furthermore, Figure 4 displays the SINR model using equa-

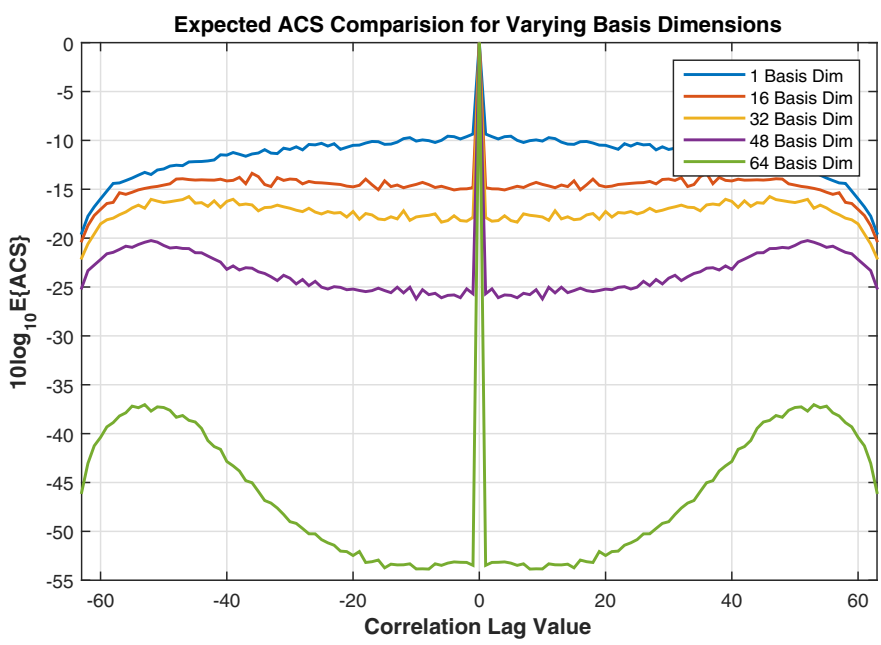

Fig. 6. Expected ACS for progressively increasing signal basis dimensions over 100 Monte Carlo trials.

tion (15) and shows good performance (small mismatch) for higher dimensions but a more conservative estimate for smaller basis. While we consider this a good first order approximation of SINR degradation, additional investigations are necessary to provide a more accurate model.

We extend the qualitative analysis to include an investigation of the signal ACS as a function of the basis dimension. As expected, as the basis increases, the sidelobe performance improves (i.e. lower average sidelobe levels). However, the improvement is nonlinear, as shown in Figure 6 and explained in detail in section III-B.

\section{CONCLUSIONS AND Insights}

The transmit signal ISL level affects many of a radar systems core functions and the ability to understand how it affects the SINR will drive an adaptive system to place the DOF where they are most valuable, for example, in resolving targets vs. improving signal strength. In this introductory work we have developed a model for the SINR performance as a function of the eigen-basis dimension. We have also analyzed the ISL metric and identified opportunities to pursue additional analytical work to find the transition points of each zone.

We also note, this work showcases some of the challenges inherent with performance estimation (i.e. analytical intractability's, data model mismatch loss, etc.), but also allows for quick-but-rough estimates of a complicated trade-space.

Future work calls for improved analytical results. To do so, assumptions and allowances on the eigenvectors to be represented as random distributions is necessary. For example, as complex Gaussian distributed. Additional data models, including K-Distributed clutter or auto-regressive models of the interference and noise spectrum would also be appropriate for investigation.

\section{ACKNOWLEDGMENT AND DISClAIMER}

This work was supported by the Air Force Office of Scientific research under project 14RY10COR. However, the views and opinions expressed in this article are those of the authors and do not necessarily reflect the official policy or position of any agency of the U.S. government. Examples of analysis performed within this article are only examples. Assumptions made within the analysis are also not reflective of the position of any U.S. Government entity.

\section{APPENDIX:}

\section{A. Gradient of $J_{i s l}(\underline{s})$}

Given the cost function in (11) we compute the gradient vector. To facilitate the derivative operation we write (11) as a function of real variables using the following transformations

$$
\mathbb{R}\left\{\underline{\mathbf{s}}^{H} \mathbf{E}_{\mathbf{k} \underline{\mathbf{s}}\}}\right\}=\left[\underline{\mathbf{s}}_{r}^{t} \underline{\mathbf{s}}_{i}^{t}\right]\left[\begin{array}{cc}
\mathbf{E}_{\mathbf{k} r} & -\mathbf{E}_{\mathbf{k} i} \\
\mathbf{E}_{\mathbf{k} i} & \mathbf{E}_{\mathbf{k} r}
\end{array}\right]\left[\begin{array}{c}
\underline{\mathbf{s}}_{r} \\
\underline{\mathbf{s}}_{i}
\end{array}\right]
$$

and

$$
\mathbb{I}\left\{\underline{\mathrm{s}}^{H} \mathbf{E}_{\mathbf{k} \underline{\mathrm{s}}\}}=\left[\underline{\mathrm{s}}_{r}^{t} \underline{\mathrm{s}}_{i}^{t}\right]\left[\begin{array}{cc}
\mathbf{E}_{\mathbf{k} r} & \mathbf{E}_{\mathbf{k} i} \\
\mathbf{E}_{\mathbf{k} i} & -\mathbf{E}_{\mathbf{k} r}
\end{array}\right]\left[\begin{array}{l}
\underline{\mathbf{s}}_{i} \\
\underline{\mathbf{s}}_{r}
\end{array}\right]\right.
$$

where subscripts $(\cdot)_{r}$ and $(\cdot)_{i}$ denote the real and imaginary components [23]. After simplification, this allows us to write the argument in the summation of (11) as

$$
\begin{aligned}
& \left|\underline{\underline{s}}{ }^{H} \mathbf{E}_{\mathbf{k} \underline{\mathrm{s}}}\right|^{2}=\left(\underline{\mathrm{s}}^{H} \mathbf{E}_{\mathbf{k}} \underline{\mathrm{s}}\right)\left(\underline{\mathrm{s}}^{H} \mathbf{E}_{\mathbf{k}} \underline{\mathrm{s}}\right)^{H}, \\
& =\mathbb{R}\left\{\underline{\mathrm{s}}^{H} \mathbf{E}_{\mathbf{k}} \underline{\mathrm{s}}\right\}^{2}+\mathbb{I}\left\{\underline{\mathrm{s}}^{H} \mathbf{E}_{\mathbf{k}} \underline{\mathrm{s}}\right\}^{2}, \\
& =\left(\underline{y}^{t} \mathbf{B}_{1} \underline{y}\right)^{2}+\left(\underline{y}^{t} \mathbf{B}_{2} \underline{y}\right)^{2},
\end{aligned}
$$

where $\mathbf{y}=\left[\begin{array}{l}\underline{\mathbf{s}}_{r}^{t} \\ \underline{\mathbf{s}}_{i}^{t}\end{array}\right], \quad \mathbf{B}_{1}=\left[\begin{array}{cc}\mathbf{E}_{\mathbf{k} r} & -\mathbf{E}_{\mathbf{k} i} \\ \mathbf{E}_{\mathbf{k}} & \mathbf{E}_{\mathbf{k} r}\end{array}\right]$ and $\mathbf{B}_{2}=$ $\left[\begin{array}{cc}\mathbf{E}_{\mathbf{k} r} & \mathbf{E}_{\mathbf{k} i} \\ \mathbf{E}_{\mathbf{k} i} & -\mathbf{E}_{\mathbf{k} r}\end{array}\right]$. The gradient vector is simply computed 
with multiple applications of the chain rule. The complete gradient is then given as

$$
\begin{aligned}
\nabla_{\underline{\mathbf{y}}} \mathbf{J}_{\text {isl }}(\underline{y})= & 2 \sum_{k=1}^{M-1}\left(\underline{y}^{t} \mathbf{B}_{1} \underline{y}\right)\left(\mathbf{B}_{1}+\mathbf{B}_{1}^{t}\right) \underline{\mathbf{y}} \\
& +\left(\underline{y}^{t} \mathbf{B}_{2} \underline{y}\right)\left(\mathbf{B}_{2}+\mathbf{B}_{2}^{t}\right) \underline{y} .
\end{aligned}
$$

\section{B. Hessian of $J_{i s l}(\underline{s})$}

The Hessian is computed using the above gradient and applying multiple applications of the chain rule. We define the Hessian as

$$
\begin{aligned}
\mathcal{H}_{\underline{y}} \mathbf{J}_{\text {isl }}(\mathbf{y}) & =\frac{\partial^{2} \mathbf{J}_{\text {isl }(\mathbf{y})}}{\partial \mathbf{y} \partial \mathbf{y}^{t}}, \\
& =\frac{\partial}{\partial \underline{y}}\left(\frac{\partial \mathbf{J}_{\text {isl }}}{\partial \underline{y}}\right)^{t}, \\
& =\frac{\partial}{\partial \mathbf{y}}\left(\nabla_{\mathbf{y}} \mathbf{J}_{\text {isl }(\mathbf{y})}\right)^{t} .
\end{aligned}
$$

Using the same definitions as above to simplify, we compute the complete Hessian as

$$
\begin{aligned}
\mathcal{H}_{\mathbf{y}} \mathbf{J}_{\text {isl }}(\mathbf{y}) & =2 \sum_{k=1}^{M-1}\left[\mathbf{y}^{t} \mathbf{B}_{1 k} \mathbf{y}\left(\mathbf{B}_{1 k}+\mathbf{B}_{1 k}^{t}\right)\right. \\
& +\left(\mathbf{B}_{1 k}+\mathbf{B}_{1 k}^{t}\right) \mathbf{y y}^{t}\left(\mathbf{B}_{1 k}+\mathbf{B}_{1 k}^{t}\right) \\
& +\mathbf{y}^{t} \mathbf{B}_{2 k} \mathbf{y}\left(\mathbf{B}_{2 k}+\mathbf{B}_{2 k}^{t}\right) \\
& \left.+\left(\mathbf{B}_{2 k}+\mathbf{B}_{1 k}^{t}\right) \mathbf{y y}^{t}\left(\mathbf{B}_{2 k}+\mathbf{B}_{2 k}^{t}\right)\right] .
\end{aligned}
$$

Of course, the Hessian can be further simplified for symmetric $\mathbf{B}_{1,2}$, allowing for ease of computation of the determinant.

\section{REFERENCES}

[1] S. Pillai, D. Youla, H. Oh, and J. Guerci, "Optimum transmit-receiver design in the presence of signal-dependent interference and channel noise," IEEE, pp. 870-875, 1999.

[2] A. M. Jones, B. Rigling, and M. Rangaswamy, "Eigen-space analysis of constrained adaptive radar waveform design," in Radar Conference, IEEE, May 2014.

[3] A. M. Jones, B. D. Rigling, and M. Rangaswamy, "Eigen-basis analysis of expected modulus perturbation for constrained signal design," in Asilomar Conference, IEEE, To Appear 2014.

[4] S. Sussman, "Least-square synthesis of radar ambiguity functions," IRE Transactions on Information Theory, pp. 246-254, April 1961.

[5] R. McAulay and R. Johnson, "Optimal mismatched filter design for radar ranging, detection, and resolution," IEEE Transactions on Information Theory, vol. 17, pp. 696-701, November 1971.

[6] J. S. Bergin, P. M. Techau, J. E. Don Carlos, and J. R. Guerci, "Radar waveform optimization for colored noise mitigation," in Radar Conference, pp. 1-6, IEEE, May 2005.

[7] L. Patton and B. Rigling, "Autocorrelation and modulus constraints in radar waveform optimization," in International Waveform Diversity and Design Conference, IEEE, 2009.

[8] P. Stoica, H. He, and J. Li, "On designing sequences with impulse-like periodic correlation,” IEEE Signal Processing Letters, vol. 16, pp. 703 706, August 2009.

[9] L. Patton, S. Frost, and B. Rigling, "Efficient design of radar waveforms for optimised detection in coloured noise," IET Radar, Sonar and Navigation, vol. 6, no. 1, pp. 22-29, 2012.

[10] S. Frost, "Performance analysis of radar waveforms for congested spectrums," Master's thesis, Wright State University, November 2011.

[11] S. Frost and B. Rigling, "Performance comparison for constrained radar waveform design,' in IEEE RadarCon 2011 Proceedings, pp. 724-728, IEEE, 2011.
[12] D. Chu, "Polyphase codes with good periodic correlation properties," IEEE Transactions on Information Theory, July 1972.

[13] P. Stoica, H. He, and J. Li, "New algorithms for designing unimodular sequences with good correlation properties," IEEE Transactions of Signal Processing, vol. 57, pp. 1415-1425, April 2009.

[14] J. Li, J. Guerci, and L. Xu, "Signal waveform's optimal under restriction design for active sensing," in Sensor Array and Multichannel Processing, 2006. Fourth IEEE Workshop on, pp. 382-386, 2006.

[15] MATLAB, Version 7.14.0.739 (2012a). The MathWorks Inc., 2012.

[16] I. Reed, J. Mallett, and L. Brennan, "Rapid convergence rate in adaptive arrays," IEEE Transactions on Aerospace and Electronic Systems, vol. 10, pp. 853-863, November 1974.

[17] S. Boyd and L. Vandenberghe, Convex Optimization. Cambridge University Press, 2004.

[18] S. Kay, Fundamentals of Statistical Signal Processing: Detection Theory. Upper Saddle River, New Jersey: Prentice Hall, 1998.

[19] S. Haykin, Adaptive Filter Theory. Upper Saddle River, New Jersey: Prentice Hall, third ed., 1996.

[20] J. R. Guerci, R. W. Schutz, and J. D. Hulsmann, "Constrained optimum matched illumination-reception radar," United States Patent, p. 8, September 1992.

[21] V. Marcenko and L. Pastur, "Distribution of eigenvalues for some sets of random matrices," Math USSR-Sbornik, vol. 1, no. 4, pp. 457-483, 1967.

[22] A. Gray and J. Markel, "A spectral-flatness measure for studying the autocorrelation method of linear prediction of speech analysis," IEEE Transactions Acoust. Speech Signal Processing, vol. 22, pp. 207-217, 1974.

[23] A. Beck and Y. Eldar, "Strong duality in noncovex quadratic optimization with two quadratic constraints," SIAM Journal On Optimization, vol. 17 , no. 3 , pp. $844-860,2006$. 